\title{
PERCEPCIONES DE EQUIDAD E IGUALDAD DE GÉNERO EN ESTUDIANTES DE PEDAGOGÍA ${ }^{1}$
}

\author{
Ilsa MENDOZA MENDOZA ${ }^{2}$ \\ Susan SANHUEZA HENRÍQUEZ ${ }^{3}$ \\ Miguel FRIZ CARILLO ${ }^{4}$
}

\section{Resumen}

El presente estudio analiza las percepciones que tienen los estudiantes de pedagogía sobre equidad e igualdad de género a través de un enfoque cualitativo. Los resultados evidencian desigualdades de género propias de una sociedad patriarcal que impone estereotipos y promueve lo masculino por sobre lo femenino. En este contexto, la educación aparece como facilitador de oportunidades para prácticas de igualdad y equidad de género.

Palabras Clave: Género, igualdad de género, equidad de género, percepciones, educación superior.

\footnotetext{
${ }^{1}$ El artículo se enmarca en el proyecto Inmigrantes en el sistema educacional de Chile: Representaciones de género, lengua, territorialidad y exclusión social, financiado por el programa de investigación asociativa ANILLOS DE INVESTIGACION EN CIENCIAS SOCIALES Y HUMANIDADES de CONICYT (2015-2018), Numero SOC 1401.

2 Ilsa Mendoza Mendoza: Dra en Educación, Universidad Católica del Maule (UCM). Su principal línea de investigación es Educación, Migración e Interculturalidad.

Email:2004.mendoza@gmail.com
}

3 Susan Sanhueza Henríquez: Doctora en investigación educativa, Directora Centro de Estudios Migratorios e Interculturales y del Doctorado en Educación de la Facultad de Ciencias de la Educación, Universidad Católica del Maule. Su principal línea de investigación es Migración y Educación Intercultural, Comunicación Intercultural. Email: ssanhueza@ucm.cl

Publicaciones: Understanding elementary and secondary students representation of cultural differences as reflected in the process of intercultural communication in school contexts, Nordic Psychology, 67(1), 6586. 2015 (ISI).

The Intercultural Sensitivity of chilean teachers serving an im- migrant population in schools. New Approaches in educational Research, 5(2), 131-156. 2016 (ISI).

\footnotetext{
${ }^{4}$ Miguel Friz Carillo: Doctor en Ciencias matemáticas, Académico y Director del Departamento de Ciencias de la Educación, Universidad del Bío-Bío (UBB) y grupo de Investigación en educación y Educación Matemática. Su principal línea de investigación es Didáctica de las Matemáticas, Migración y Educación, Comunicación Intercultural. E-mail: mfriz@ubiobio.cl

Publicaciones: Competencias comunicativas interculturales en la formación inicial docente: el caso de tres universidades regionales de chile, Estudios Pedagógicos Scopus Aceptada Chile, 2016.

Estudio exploratorio sobre actitudes y comportamiento del profesorado de chile en contextos de escolarización del alumnado inmigrante. Revista Electrónica de Investigación Educativa, Vol.16, Número 3 México.2014
} 


\begin{abstract}
This study analyzes the perceptions student teachers on gender equity and equality through a qualitative approach. The results show gender inequalities inherent in a patriarchal society that imposes stereotypes and promotes the masculine over the feminine. In this context, education appears as a facilitator of opportunities to practice gender equality and equity.
\end{abstract}

Keywords: Gender, gender equality, gender equality, perceptions, higher education.

Résumé: Cette étude analyse les perceptions des enseignants des étudiants sur l'égalité des sexes et de l'égalité grâce à une approche qualitative. Les résultats montrent les inégalités de genre inhérentes à une société patriarcale qui impose des stéréotypes et favorise le masculin sur le féminin. Dans ce contexte, l'éducation apparaît comme un facilitateur des occasions de pratiquer l'égalité des sexes et de l'équité.

Mots clés: Le sexe, l'égalité des sexes, l'égalité des sexes, la perception, l'enseignement supérieur.

\title{
INTRODUCCION
}

Históricamente a partir de la estructura corporal biológica, es decir, del sexo con el cual se nace, la sociedad ha asignado a los individuos el rol de hombre o mujer y en base a ello las características, actitudes y comportamientos que se espera según su sexo. Bajo estas categorías binarias, si se nace biológicamente hembra, se es mujer y se pertenece al género femenino y si se nace biológicamente macho, se es hombre y se pertenece al género masculino. Esta lógica cultural dicotómica sobre la cual se ha ordenado la sociedad, ha dado lugar a concepciones y percepciones locales e históricas sobre lo que es propio tanto de hombres, como de mujeres, (Lamas, 1996) conformando con ello estereotipos discriminadores, que han obstaculizado la igualdad de oportunidades entre ambos sexos (Godoy y Mladinic, 2009).

Desde esta perspectiva cobra importancia analizar y evaluar las concepciones y percepciones de equidad e igualdad de género desde el escenario educativo, considerando el rol que tiene la educación en la producción y transmisión del conocimiento validado socialmente. Por lo que se convierte en un espacio poderoso para el desarrollo, comprensión y revalorización equitativa de las categorías existentes de sexo y género, pudiendo contribuir para que mujeres y hombres convivan en ambientes de respeto y en condiciones de igualdad mutuas (Rebolledo, 2015). Más aún, cuando diversos estudios han señalado al espacio educativo como el fortalecedor de una cultura androcéntrica, tanto en su curriculum formal como en su curriculum oculto (Gore, 1996; Díaz Rodríguez, 2003; López-Navajas, 2014).

En este sentido, en el caso de Chile el principio de autonomía de enseñanza del sistema educacional ha posibilitado que los temas de género queden supeditados a la libre elección de los proyectos educativos que cada institución determina, debido a que los mecanismos de acreditación de la calidad establecidos en el país no poseen la facultad legal de revisar las mallas curriculares en términos de sus contenidos y objetivos de aprendizaje en educación superior, sino solo de su coherencia interna. De modo tal, que si bien algunas universidades han incorporado en sus mallas curriculares cursos de género, estos han sido incorporados transversalmente como unidades temáticas, o han tenido un carácter de optativos. Este hecho ha evidenciado un déficit de conocimientos y habilidades tanto entre los (as) docentes, los (as) futuros (as) docentes, como en los (as) formadores de formadores (Caviedes et al., 2006).

En consecuencia, estudiar las percepciones que tienen los estudiantes de pedagogías en las universidades como espacios públicos generadores de conocimientos, ayudara a la discusión de la proyección de políticas educativas que consideren la perspectiva de género en el sistema educativo chileno, en concordancia con su responsabilidad en la promoción del respeto por la diversidad, la tolerancia y las diferencias de género de las personas. Para ello el artículo comienza con la diferenciación de género y sexo constituido socialmente, lo cual permite evidenciar construcciones desiguales que han posicionado al hombre en un nivel de superioridad 
respecto a la mujer, dando paso a una serie de estereotipos y desigualdades sociales. En segundo lugar y desde un enfoque inspirado en la teoría fundamentada se dan a conocer los principales resultados de las percepciones subjetivas que tienen los estudiantes del fenómeno en estudio. Finalmente y a partir de los resultados se presentan las conclusiones y se discute la necesidad de la elaboración de políticas educativas que propicien el desarrollo de un currículo con perspectiva de género en las universidades chilenas.

\section{La distinción y construcción de género/sexo}

La psicología médica fue la primera disciplina que considero las características sociales y culturales para diferenciar al género del sexo, concluyendo que el sexo hace referencia a rasgos fisiológicos y biológicos de ser hombre o mujer, en tanto el género es la construcción social de esas diferencias sexuales (Stoller, 1968). De este modo, se estableció que el género es una construcción social que asigna al hombre y a la mujer determinadas características psicológicas y sociales que una cultura atribuye para cada uno de ellos, lo cual se traduce entre otras cosas en prácticas, representaciones, normas, (Lamas, 2015), que junto con producir diferencias entre el género masculino y femenino, construye también diferencias que involucran desigualdades y jerarquías entre hombres y mujeres en sociedades marcadamente androcéntricas (Burín, 1998). A partir de estas construcciones desiguales preestablecidas socialmente se ha posicionado al hombre en un grado de superioridad frente a la mujer, lo que ha llevado a considerar a estas últimas como el sexo débil asociado a la sumisión, el orden o pasividad, mientras que lo masculino se lo ha relacionado con la virilidad, competitividad, agresividad e independencia. Propiciando así un contexto para la justificación de la dominación de los hombres sobre las mujeres (Bourdieu, 2000).

Estas transmisiones y conductas esperadas culturalmente se han dado en todos los espacios de interacción en los cuales transcurre la vida de la persona, siendo las más importantes la familia y la escuela. Así, durante los primeros años de vida -incluso antes del nacimiento- la socialización la realizan los padres a partir del sexo biológico, atribuyendo características estereotipadas adscritas a un género femenino o masculino, tales como vestimentas, comportamientos, actitudes, expectativas, tareas, obligaciones, etc., (Méndez, 2014). Luego es la institución educativa como espacio socializador, la encargada de que hombres y mujeres aprendan las formas de actuar deseadas y aceptadas socialmente, transmitiendo así saberes valorados y legitimados por cada tipo de sociedad. Es a través del currículum que el sistema educativo selecciona los saberes que se espera que las nuevas generaciones adquieran para reproducir el orden social establecido (Abett de la Torre, 2014).

En este sentido, diversos estudios han revelado que en la práctica pedagógica se reproducen patrones identitarios discriminadores, como por ejemplo el uso generalizado de un lenguaje sexista que establece el genérico masculino invisibilizando al género femenino, materiales educativos androcéntricos con ausencia de la mujer en un $70 \%$ de los casos, expectativas diferenciadas de parte de los profesores según el sexo, formación científica a favor de los niños, etc., (Moreno, 1986; Díaz, 1999; Bernal, 2007). Ante estas evidencias y considerando que género al igual que curriculum es una construcción social, es que este último debe ser comprendido de una manera amplia, tanto en lo explicito como lo implícito, debido a que como construcción social puede ser un espacio transformador de conductas y creador de nuevas formas culturales en la medida puede ser modificado. Para lo cual es necesario que se aborden las construcciones culturales tradicionales sobre lo que es ser hombre y ser mujer desde la formación y el accionar del profesorado, considerando la importancia que los profesores tienen en el proceso formativo del ser humano. Más aún cuando este ha tendido a reproducir una cultura de género androcéntrica, en la que lo masculino ha dominado y oprimido a lo femenino, negándole oportunidades y limitando su accionar a lo socialmente normado rigiendo la convivencia entre hombres y mujeres (Bourdieu, 2000) en condiciones sociales desiguales. 


\section{Estereotipos y desigualdades sociales}

La dicotomía existente entre lo masculino y lo femenino -con sus diferencias culturales- ha establecido estereotipos sociales que determinan los roles y limitan las potencialidades de las personas bajo la concepción de que hay ciertas capacidades, emociones y conductas que corresponden diferenciadamente a hombres y mujeres. Estos estereotipos sociales se manifiestan en los distintos espacios de la vida, entre ellos en la televisión y en el espacio laboral. La publicidad televisiva con el propósito de lograr cumplir con las metas de mercado, exagera ciertas cualidades establecidas socialmente provocando en muchos casos fenómenos distorsionados y ajenos a la realidad. Así la mujer continua siendo representada como madre y esposa, conservando los roles tradicionales que culturalmente se le han impuesto y que la sitúan en una posición de dependencia respecto al hombre, en tanto este último es representado como el exitoso, el patriarca, el proveedor, etc., conservando la superioridad que se le ha asignado socialmente respecto a la mujer (Fajardo, 2007; Conde y del Hoyo Hurtado, 2011; Barrios, 2011). Por su parte en el espacio laboral la función asignada naturalmente a lo femenino es la reproductora y la crianza de los hijos, mientras que a lo masculino le asigna la manutención familiar, esto es, el espacio público y productivo (Richter, 2007).

En esta línea y si bien en el caso de Chile, la participación de la mujer en la fuerza laboral ha presentado un aumento sostenido en el tiempo alcanzando en el último trimestre del año 2013 un 48,3\%, esta continua siendo inferior a la tasa de participación de los hombres en el mercado laboral, la cual alcanza un $72 \%$ durante el mismo periodo (Instituto Nacional de Estadística de Chile, 2015). Estos datos evidencian la persistencia de procesos de discriminación que imponen una serie de obstáculos para el desarrollo de las trayectorias laborales de las mujeres en igualdad de condiciones respecto de los hombres, ubicándolas generalmente en posiciones jerárquicamente inferiores relacionadas con el desarrollo de roles familiares, tales como el cuidado infantil y las labores domésticas, asociadas a rasgos o disposiciones internas, como calidez, amabilidad y empatía. A diferencia de los rasgos atribuidos al hombre como asertividad, autonomía, independencia que los posiciona en roles laborales de mayor estatus y poder (Godoy y Mladinic, 2009).

Estas creencias sociales estereotipadas de lo que es ser hombre y ser mujer, propician otro tipo de desigualdad que se expresa a través de la violencia de género. En Chile, según el Servicio Nacional de la Mujer (SERNAM, 2015) una de cada tres mujeres ha sufrido algún tipo de violencia de parte de alguna de sus parejas, y estudios recientes indican que la violencia se inicia en las relaciones de noviazgo, evidenciando la presencia y reproducción de patrones culturales sexistas (Valdivia y González, 2014; Gómez et al., 2015; Rubio-Garay et al., 2015) relacionados con asociaciones conscientes e inconscientes respecto de las conductas de las personas en base a su sexo socialmente identificado. Ante estas concepciones estereotipadas es que la educación, como ya hemos mencionado anteriormente, juega un rol clave para una comprensión adecuada del concepto de género, en la medida que posibilita razonar que lo que pensamos que son atributos específicos de hombres o de mujeres, en realidad son características construidas socialmente que no tienen relación con lo biológico, puesto que esto no es suficiente para provocar características o comportamientos atribuibles exclusivamente a un determinado sexo. Esto debido a que tanto mujeres como hombres, comparten rasgos y conductas humanas (Lamas, 2015), por lo tanto se convierte en un espacio posibilitador para la comprensión de que ser hombre y ser mujer es un dato cultural y no biológico, pudiendo transformar los prejuicios de género establecidos socialmente.

En este contexto, el presente estudio pretende analizar las percepciones que tienen los (as) estudiantes de pedagogías de último año de formación en relación a la equidad e igualdad de género. En específico, a) conocer las representaciones de género, b) identificar los temas académico-pedagógicos relativos a género que han recibido los (as) estudiantes en su formación profesional, c) conocer la percepción sobre situaciones cotidianas de discriminación por género y d) analizar la relación género y oportunidades laborales de hombres y mujeres. 


\section{MÉTODO}

\section{Enfoque y diseño}

Hemos optado por una metodología de investigación cualitativa, con un enfoque inspirado en la teoría fundamentada de Glaser y Strauss (1967) y los trabajos posteriores de Strauss y Corbin (2002), los cuales la definen como un método que intenta construir teorías explicativas de fenómenos sociales, a partir del levantamiento de datos empíricos que son sistemáticamente analizados durante el proceso de exploración. En consecuencia, a partir de las percepciones subjetivas que tienen los estudiantes se accede a los significados que estos le atribuyen al fenómeno en estudio, intentando desarrollar un conjunto de conceptos que al integrarse, proveen una explicación teórico-descriptiva. Dado que este estudio se centró en las percepciones subjetivas de los estudiantes, se optó por no ofrecer una definición a priori del fenómeno en estudio, quedando la definición abierta a la conceptualización de los estudiantes, como una forma de contextualizar su mirada y facilitar su propio discurso.

\section{Contexto y participantes}

El estudio fue realizado en una región centro-sur de Chile, región del Maule, compuesta según los datos de la última encuesta de Caracterización Socioeconómica Nacional del año 2015 (CASEN), principalmente por población rural $(34 \%)$ y de una economía silvoagropecuaria. A nivel educativo, solo existen dos universidades pertenecientes al Consejo de Rectores de Universidades Chilenas (con financiamientos del estado), desarrollando el estudio en una de ellas, la Universidad Católica del Maule. La cual tiene una matrícula cercana a los 7 mil estudiantes, siendo históricamente una institución formadora de profesores.

En particular, optamos por un muestreo intencionado, sujeto a disponibilidad (Cardona, 2002) con la participación de cinco estudiantes de pedagogía. Nos planteamos como criterios de participación la diversidad de género, diferentes titulaciones, educación básica, educación media y de diferentes edades, estableciendo además el criterio de "estudiantes en último año de formación" bajo el supuesto que ellos deberían contar con una comprensión más profunda de la temática por encontrarse próximos a insertarse en contextos laborales, donde se espera puedan desarrollar prácticas inclusivas en materia de género.

\section{Técnicas de recolección de datos}

La técnica de recolección de información fue la entrevista semiestructurada, la cual intenta comprender más que explicar, bajo el marco de respuestas abiertas sin categorías preexistentes, donde el entrevistador sigue un esquema general y flexible de preguntas, en cuanto a orden, contenido y formulación de las mismas. Este tipo de entrevista concibe al actor social, como una persona que construye sentidos y significa la realidad a través de un marco complejo de creencias y valores, construido con otros, pero irreductiblemente único para él (Ruiz, 2007).

\section{Procedimiento y análisis de datos}

Las entrevistas se realizaron en la región del Maule, en la ciudad de Talca correspondiente al segundo semestre del año 2016. Las entrevistas fueron transcritas del mismo modo como fueron respondidas por los estudiantes y los códigos utilizados corresponden al entrevistado seguido del número y de la pregunta correspondiente, por ejemplo, $\mathrm{E}=$ Estudiante, $\mathrm{p}=$ pregunta, con su respectivo número. En los casos en los cuales de una misma pregunta se deriven otras preguntas, estas se acompañarán de letras $=\mathrm{a}, \mathrm{b}, \mathrm{c}$.

El análisis de la información se realizó según el método de comparación continua de Strauss y Corbin (2002), lo cual se tradujo en procedimientos sistemáticos de codificación con la intención de sistematizar propiedades e hipótesis acerca del fenómeno en estudio a partir del principio de la saturación teórica (Glaser y Strauss, 1967). De este modo, la primera fase, de categorización abierta, permitió obtener categorías descriptivas de información a través de un doble proceso de fragmentación y agrupación de los datos provenientes de las entrevistas. 
Luego las categorías emergentes fueron progresivamente integradas y contrastadas en el proceso de codificación axial, arrojando un total de cuatro categorías temáticas, que fueron agrupadas y que sirvieron de vinculo de los diferentes contenidos relacionados con el objetivo de investigación. Por último, el proceso de codificación selectiva integró estas categorías principales a partir de una relectura de los datos, obteniéndose un esquema teórico mayor.

\section{RESULTADOS}

\section{Construcción de género}

Esta categoría grafica las ideas y representaciones sociales que tienen los (as) estudiantes relativas a género y que están relacionadas al primer objetivo específico del presente estudio.

\section{Figura 1}

\section{Nociones de género de los estudiantes}

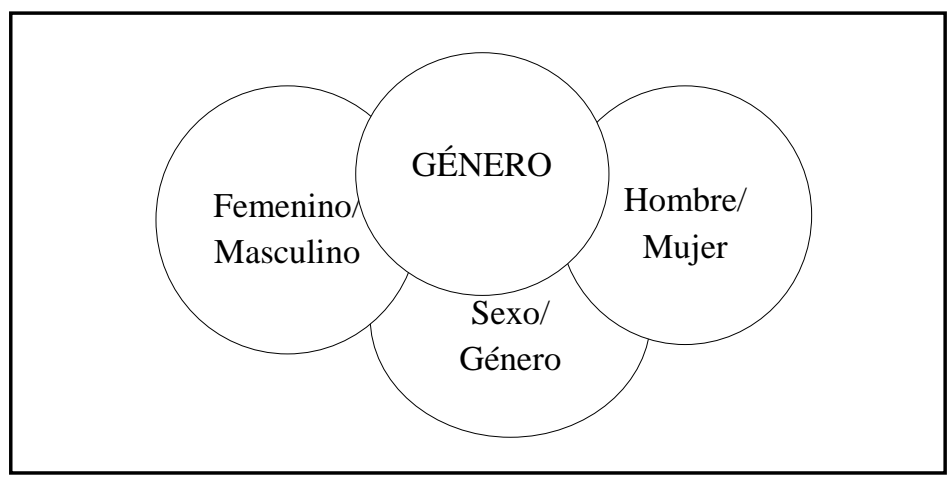

Fuente: elaboración propia

Las ideas que subyacen a lo que los (as) estudiantes entienden por género, es la concepción binaria de sexo/género: hombre/mujer; femenino/masculino. De este modo, cuando se les interroga a los (as) estudiantes de lo que entienden por género, ellos (as) manifiestan lo siguiente:

"Genero hombre y mujer eso entiendo por género". (E2, p1).

“(...) se podría decir, como catalogar hombre mujer (...), como el sexo para diferenciar una persona su género masculino o femenino”. $(E 3, p 1)$.

“(...) es como el sexo, relación de hombre y mujer”. (E4, p1).

Como se puede observar en las citas anteriores, los (as) estudiantes entienden por género la concepción binaria de los sexos, la diferenciación entre hombre y mujer dada por lo biológico. Estas ideas contienen la asignación estereotipada de los roles preestablecidos y construidos socialmente de lo que significa ser hombre y ser mujer a partir de la diferencia anatómica, lo cual puede obedecer en parte, a la generalización que se ha originado en español del uso del término para indicar al hombre como "género masculino" y a la mujer como "género femenino" sin que ello exprese necesariamente, la comprensión de la lógica cultural de género (Lamas, 2015). De este modo, el género es conceptualizado como un atributo que permite clasificar a los individuos entre hombres y mujeres, no como una construcción social. 
Bajo estas comprensiones de un sistema de géneros binarios, permanece la creencia que el sexo refleja el género, es decir, no existe una independencia de uno y otro, sino una correlación, obviando el hecho que si bien el origen biológico establece diferencias anatómicas entre hombres y mujeres, esto no es indicativo de un comportamiento exclusivo de un sexo. Por lo cual el género no puede reducirse a la comprensión biológica dicotómica hombre/mujer, sino que es necesario considerar los condicionamientos sociales que intervienen en dicha construcción de género, debido a que nada asegura que alguien que nace mujer corresponda al género "femenino" o viceversa (Butler, 1997).

Estas ideas convencionales de ser hombre y ser mujer, de lo femenino y masculino, de sexo y género que se revelan en las respuestas de los estudiantes, se relacionan con las construcciones sociales legitimadas a través de la historia por las diversas culturas, las cuales han asignado aspectos psicológicos y culturales para determinar el género, y han limitado el sexo a las características anatómicas y fisiológicas que distinguen al macho y la hembra en la especie humana (Aguirre y Obach, 1999). Sin la consideración que el género se constituye de distintas formas dependiendo de los distintos contextos socioculturales, y que no es algo dado por las características biológicas. De este modo, los (as) estudiantes responden a la concepción tradicional de género, binaria y dicotómica, esto es, la consideración del sexo relacionada con ser hombre o mujer, femenino o masculino. Esta concepción de diferencias biológicas y fisiológicas que se identifican con un sexo biológico y que determinan las distinciones sociales entre lo femenino y masculino, ha sido reforzado por una cultura patriarcal que ha dirigido su atención a la subordinación de las mujeres y la necesidad de dominio del hombre sobre la mujer (Scott, 2008).

En este escenario es que la educación como constructora de cultura, puede reproducir o transformar formas de pensamiento y acción social a través del curriculum. El cual como construcción social e histórica establece significados, estereotipos, valores, creencias, normas, etc., que estructuran las formas a través de las cuales las personas y las instituciones se relacionan y gobiernan entre sí, interpelando la conciencia de quienes componen una determinada comunidad (Guerrero et al., 2006).

\section{Déficit en la formación pedagógica}

El desarrollo de una sociedad más equitativa e igualitaria implica una transformación de los valores y creencias con respecto al género y las relaciones de género existentes (Lagarde, 1996), para lo cual se requiere del compromiso del profesorado como agente clave en la renovación de la cultura de género, que aporte nuevos significados y códigos en la práctica. En este sentido, la axial número dos nos permite identificar los temas académico-pedagógicos relativos al género que han recibido los (as) estudiantes en su formación profesional, y que corresponden al segundo objetivo específico del estudio.

\section{Figura 2}




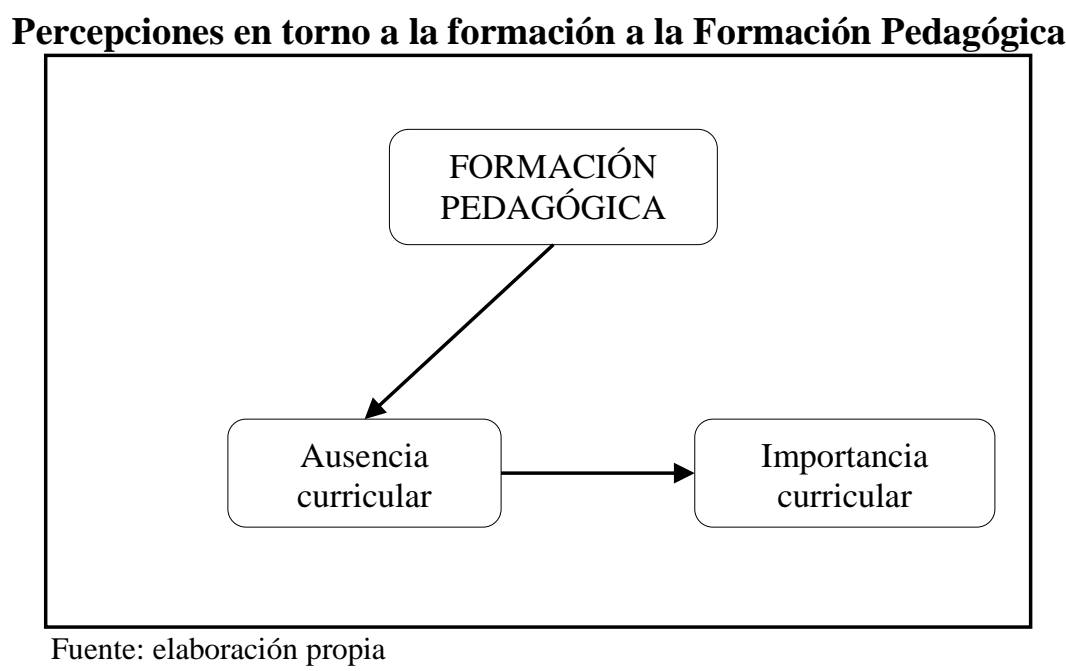

Los (as) estudiantes expresan que no existe en su malla curricular un curso o unidad que aborde la perspectiva de género, por lo tanto, las ocasiones en que el tema ha sido abordado ha obedecido a circunstancias específicas y con profesores puntuales que trabajan desde la transversalidad en sus disciplinas de estudios y no a la incorporación del tema en su formación pedagógica.

“Género en ¿sí? O sea en ramos de educación (...) hemos visto el tema de género, igualdad de género y sexualidad (...) una de las unidades era el tema de la sexualidad, (...) hubo un debate, (...) fueron como dos sesiones de lo mismo (...)". (E1, p16).

"No, que yo recuerde no". (E2, p16).

"Curso asi ¿especifico? No, pero si hemos tratado el tema hartas veces sobre todo en los ramos que son de psicología el desarrollo de la niñita el desarrollo del niñito yo creo que ahí hemos tratado el tema de género, por ese lado”. (E3, p16).

Esta ausencia del tema género en su proceso formativo indica que el conocimiento que ellos poseen de género lo han adquirido en el seno familiar, con los amigos, en la vida, etc., lo cual tiende a reproducir concepciones culturales que asocian el tema género con lo biológico, específicamente con el desarrollo biológico y sexual tal como lo indica el estudiante 3 (E3), reforzando con ello una concepción tradicional y binaria del término. La ausencia curricular de la perspectiva de género en su proceso de formación de profesores, merma la posibilidad de desarrollar una visión que pueda ir más allá de las diferencias biológicas que puedan existir entre hombres y mujeres, que se englobaría en el concepto "sexo". De ahí que los (as) estudiantes manifiestan la importancia y necesidad de la incorporación de la perspectiva de género en su proceso formativo, para así poder contar en su actividad docente con los conocimientos cognitivo y conceptual de los elementos básicos para abordar e instruir a sus alumnos (as) fundamentadamente, desde una visión elaborada de género que les permita distinguir entre la diferencia sexual y las atribuciones, ideas y representaciones sociales que se construyen considerando dicha diferencia. De modo tal de poder contribuir en la igualdad y equidad de género desde la diferencia, bajo la fundamentación de la importancia que tienen tanto hombres como mujeres dentro de la sociedad.

\section{Sociedad normada y discriminadora}

La axial número tres nos permite conocer la percepción de los estudiantes sobre situaciones cotidianas de discriminación por género y que corresponde al tercer objetivo del presente estudio. 


\section{Figura 3}

\section{Percepciones de discriminación por género}

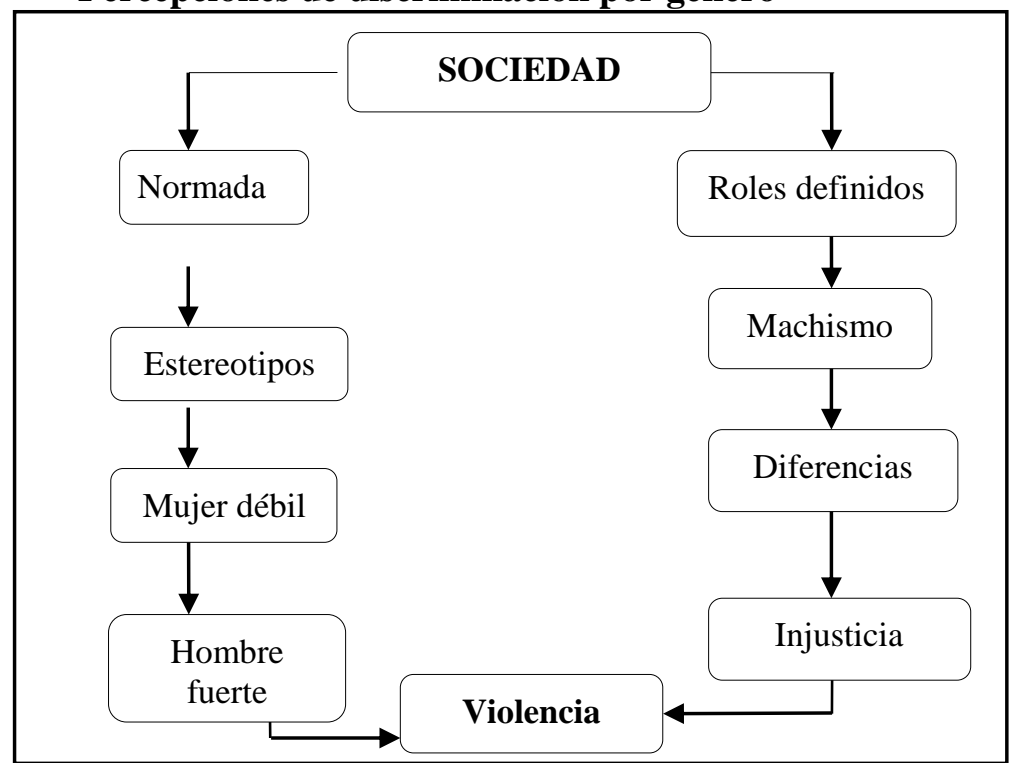

Fuente: elaboración propia

Los (as) estudiantes expresan que existen roles definidos para hombres y mujeres que se traducen en la vida cotidiana en inequidades y desigualdades normadas y validadas socialmente. Estas situaciones guardan directa relación con los estereotipos asociados al sexo biológico de las personas que la sociedad establece, y que es reproducido en los distintos ámbitos de interacción social.

"La sociedad lo define, la sociedad y las mismas personas en la casa lo definen. (...) entonces si la mamá llega y dice estas tareas son de la mamá o tiene que hacerla solamente las mujeres, obviamente se hace un traspaso cultural de lo mismo". (E1, p22).

“(...) las mujeres como siempre han sido las que hacen las cositas, igual los hombres se aprovechan de eso y nosotros no hacemos nada. Las cosas de la casa las hace mi mamá en mi lugar, las cosas que son ya más ordenar cosas, cargar cosas, arreglar algo, ahí más pega pa' hombres". (E2, p22).

"Cuando es de naturaleza física biológica yo creo que sí, (...) lo que es en los detalles, lo que es la estética, la belleza, la mujer de todas maneras y yo creo que eso va a seguir porque es un tema de naturaleza". (E3, p22).

Las expresiones anteriores refuerzan la idea de asignación de roles y conductas esperadas a partir del sexo biológico. Esta asignación se produce principalmente a juicio de los (as) estudiantes, en el seno familiar, en donde es la madre la que generalmente designa estos roles a partir de lo que la sociedad establece dependiendo del sexo biológico, lo cual es aceptado por los hombres de modo natural según el orden social preestablecido. Esta diferenciación en la asignación de funciones conlleva la presencia de relaciones de poder desiguales y dominantes (Bourdieu, 2000), las cuales son reproducidos en los distintos ámbitos de la sociedad, como es el caso de la publicidad. Así, cuando se pregunta a los (as) estudiantes si consideran que los medios de comunicación social estimulan los estereotipos de género ellos responden:

"Sí, mucho (...) la mujer tiene que ser delgada, (...) tiene que estar pendiente (...) de los hijos, porque nunca se ha visto un comercial donde el papá cuide a los hijos, de que el papá le sirva la comida, (...) el hombre los roles del trabajo, el hombre siempre está cansado trabajando (...) en cambio la mujer tiene que estar siempre al cuidado de los hijos" (E1, p23). 
"Yo siempre he tendido a pensar que la sociedad es muy machista, muy machista entonces todo lo que hace el hombre aunque lo haga mal es hombre y por eso se puede equivocar (...) por el tema de la crianza, yo siempre he pensado de que el tema de la crianza hace a las personas machistas o no machistas (...). Y eso si lo agregamos a una sociedad que sea muy estructurada, de que el hombre tenga mayor desarrollo social que la mujer, que la mujer no pueda optar a ciertas cosas a pesar de que estamos en el siglo XXI” (E1, p23).

Las diferencias de género son estimuladas por la publicidad, la familia y la sociedad en general a juicio de los (as) estudiantes. De este modo lo masculino continua siendo representado como superior ante lo femenino, caracterizando a los hombres como personas importantes, autónomas, fuertes, potentes, racionales, emocionalmente controladas, proveedores de la familia, en oposición a las mujeres, a quienes se las concibe, como débiles, emotivas, no controladas emocionalmente, al cuidado de los hijos, restringiendo su rol al ámbito doméstico. Estas concepciones atribuidas al género femenino son uno de los factores explicativos de la violencia hacia la mujer (Soto-Quevedo, 2015). Los (as) estudiantes perciben que el hombre al saberse proveedor siente que tiene toda la autoridad y poder para exigir ciertas conductas de la mujer, lo que en oportunidades convergen en situaciones de violencia. Sin embargo, ellos se declaran totalmente en contra de cualquier conducta violenta en las relaciones de pareja, pero están conscientes de que es una realidad cada vez más frecuente y cercana, en especial la violencia psicológica, de la cual han sido testigos entre sus amigos, compañeros y en un caso protagonistas.

Desigualdad de oportunidades

La axial número cuatro agrupa las condiciones que influyen en las diferentes oportunidades laborales y de ascenso profesional que la sociedad ofrece a hombres y mujeres.

\section{Figura 4}

\section{Género y oportunidades laborales}

Analizar la relación género y oportunidades laborales de hombres y mujeres

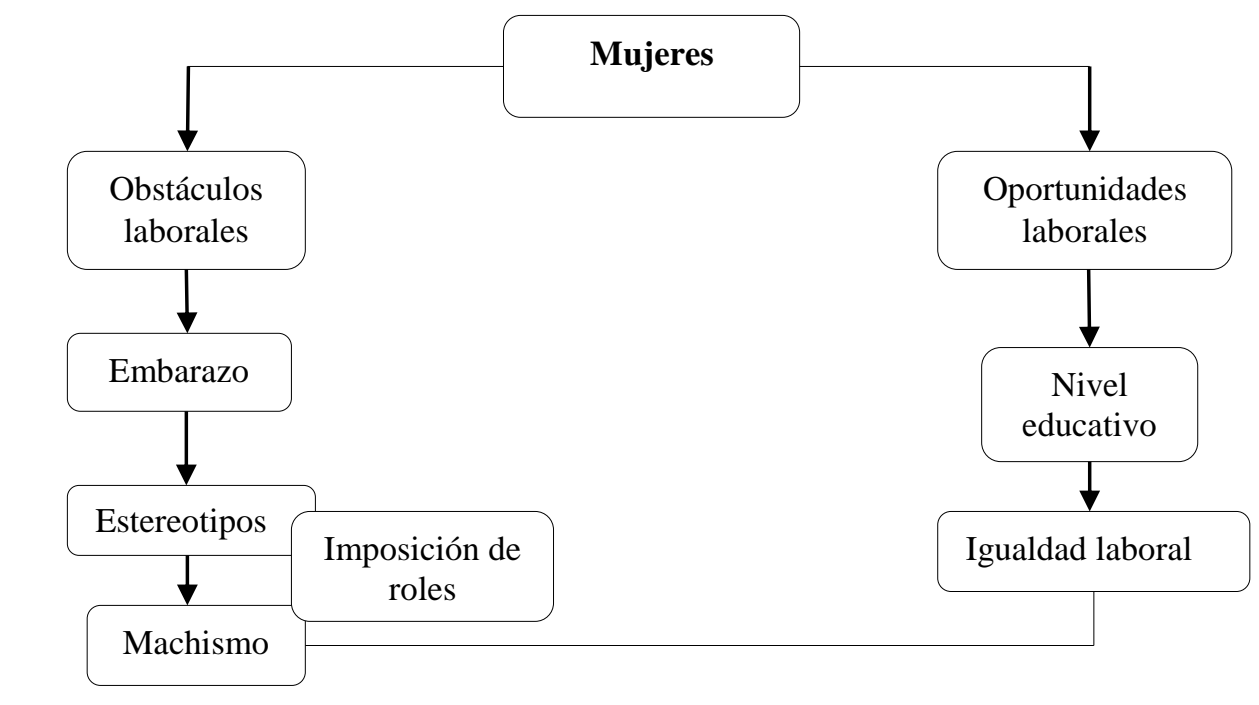

Fuente: elaboración propia 
Uno de los mayores obstáculos que visibilizan los (as) estudiantes entre las diferentes oportunidades que tienen hombres y mujeres en el mercado laboral, es el embarazo y lo que esto conlleva. Es decir, uso del pre y postnatal para la mujer, licencias en caso de enfermedad del hijo, etc., es interpretado como una perdida para el empleador en meses de producción que no son compensados, por lo tanto no le "conviene" contratar a una mujer.

“(...) tengo una compañera que fue mamá hace poco, a ella le dieron un beneficio de postnatal, y no sé qué problema tuvo pero ella estaba aburrida del trabajo y se quería cambiar a otro y como que no la llamaron, y le ha pasado en varias "pegas" por asi decirlo (...) y ella piensa que es por el bebe y yo también pienso que sí (...). Yo me pongo en el lugar del empleador a mí no me conviene contratar una mujer y póngase usted si se embaraza o tiene una guagüita y va a tener inconvenientes para venir al turno y todo eso, mejor contratar a un hombre no me va a dar problema en eso, es como pucha súper injusto pero obviamente como todo es dinero hoy en día, es lamentable" (E3, p27).

En lo que respecta a las dificultades que enfrentan las mujeres para ascender laboralmente, los (as) estudiantes lo asocian exclusivamente a situaciones de machismo que imperan en la sociedad y que continúan situando a la mujer como el sexo débil y no capacitada para desarrollar cargos de jefatura.

“(...) cuando la mujer ocupa un cargo superior o es la cabeza de algo siempre se va a pensar de que la mujer va a cometer muchos errores o de que no va a ser capaz de lograrlo" (...)" $(E 1, p 29)$.

"El trato quizás de sus compañeros con ella (...) Yo creo que a los hombres no les gusta mucho tener una jefe mujer. Así por el hecho de ser mujer simplemente. Yo creo que lo ven como el sexo débil, así lo verán y que mi jefa es del sexo débil como se ve en la sociedad el sexo débil de la mujer" (E2, p29).

Los estereotipos asociados a cada sexo vuelven a aparecer cuando se trata de posiciones de jerarquía laboral. Sin embargo, llama la atención las diferentes percepciones que tienen los y las estudiantes respecto del ejercicio de jefaturas de parte del sexo femenino, así mientras los estudiantes consideran que "Otros", no ellos, pueden considerar a la mujer débil para ese tipo de cargos y que los hombres se pueden sentir incomodos al ante una jefatura femenina, las estudiantes sienten que las mujeres son competentes y pueden desarrollar mejor que los hombres los cargos de autoridad. El consenso entre las distintas percepciones de los (as) estudiantes viene por el nivel educativo, el cual a juicio de ellos, es un factor que reduce la desigualdad de oportunidades laborales:

“(...) yo creo que los empleadores ven más capacitados a los hombres para desempeñar la labor, la desempeñan más rápido, eso verán los empleadores pero solamente en las personas que no tienen estudios. Porque las personas que tienen estudios, yo creo que ahí cambia drásticamente el tema y yo creo que ahi están súper equiparados” (E2, p10c).

En cada una de las citas anteriores se esconde a través de un "Otro" que se visibiliza en un "quizás", "yo me pongo en el lugar de", "yo creo que a los hombres", la construcción inconsciente que la sociedad ha establecido de la superioridad del hombre frente a la mujer.

Desigualdad e inequidad de género 
La figura cinco nos indica los resultados obtenidos en el proceso de codificación selectiva el cual integró todas las categorías principales que emergieron de la codificación axial.

\section{Figura 5}

\section{Análisis relacional de las categorías}

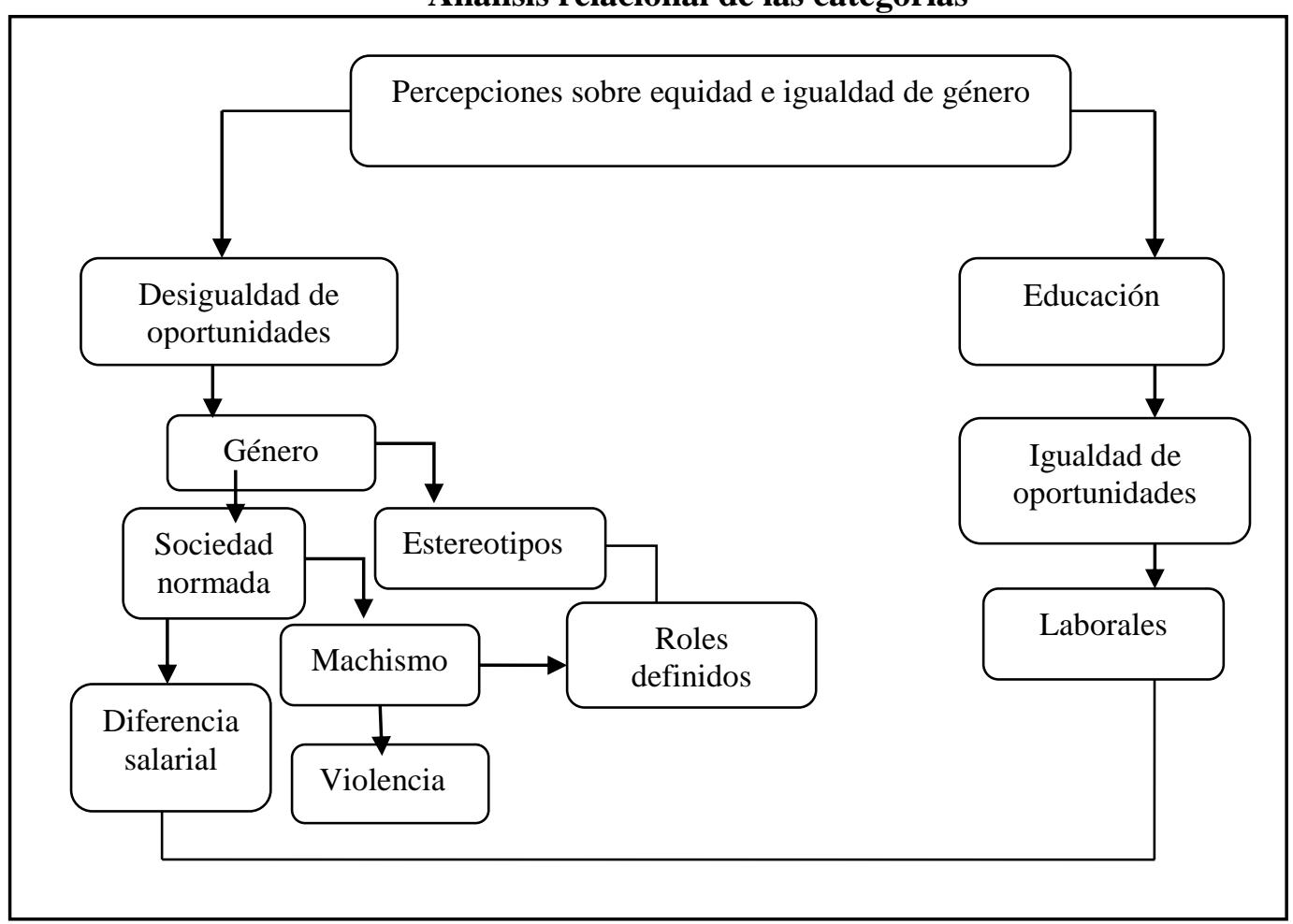

Fuente: elaboración propia

Los (as) estudiantes señalan que existen diferencias de género que favorecen a los hombres producto de una sociedad normada que construye estereotipos, en el cual el hombre es concebido socialmente como más fuerte, con voz de mando y capacidad de liderazgo, mientras que la mujer es vista como más débil y delicada, habilitada para desempeñar otras funciones. A partir de esta representación estructurada y aceptada socialmente, se establece lo que se espera que cada uno de ellos dependiendo de su sexo biológico realice, es decir, los roles de género, esto es, las actividades o tareas que se esperan que desarrollen según el sexo al que pertenece cada persona. En este sentido cuando se interroga a los (as) estudiantes si mujeres y hombres tienen los mismos deberes u obligaciones en el ámbito doméstico, laboral u otros, manifiestan la existencia de roles bien definidos socialmente:

"Los hombres tienen como el pensamiento de que la mujer tiene que cuidar a los niños, cocinar. O sea yo lo miro del ámbito de mi familia, y mi familia es súper machista, mis tías se quedan en la casa y mis tíos trabajan, ellos son los que llevan el dinero" (E5, p12).

"Socialmente se les impone que la mujer sea la que cuide a los hijos y el hombre salga a trabajar. Esas son las obligaciones que les da la sociedad a las personas, a los matrimonios. La mujer debe cumplir en la casa (...) debe hacer todo lo de la casa y además de eso, cumplir si es 
que sale a trabajar, porque si sale a trabajar y no está haciendo alguna de las dos cosas bien, debe retroceder y quedarse solo con la casa, al cuidado de los hijos. Y la obligación del padre siempre la sociedad le ha dado a cargo que tiene que salir a buscar trabajo, tiene que traer dinero y si el hombre no cumple esa obligación socialmente es mal visto" (E1, p12b).

A partir de esta distribución de funciones establecida por el sexo de las personas, se crean dos espacios de actuación y realización: el privado y el público. El privado, el del hogar, para las mujeres, por ende, los roles sociales de las mismas se enmarcan en lo doméstico: dueña de casa, madre y esposa. Mientras que el espacio público, el de las relaciones sociales con resultados en la producción, es el destinado naturalmente para los hombres, con lo cual se establece el rol de proveedor económico como su función principal, confirmando así la tradicional dicotomía entre lo público y privado, masculino y femenino respectivamente. Ante esta división en la distribución de funciones se interrogo a los (as) estudiantes si existen oficios o profesiones propias de género, lo que evidenció contradicción en las respuestas entre los diferentes sexos. Las estudiantes indicaron que el ámbito educativo y de oficios está abierto a todos por igual, no percibiendo a su juicio carreras preestablecida según el sexo biológico. Por su parte los estudiantes indican la existencia según el sexo con el cual se nace.

"No debiese pero sí existen. Por ejemplo párvulo, en párvulo la mayoría son mujeres y el hombre que entra ahi es como muy mal visto. Y la mayoría de las veces, Yo he visto que son niños gay. Mecánica también es como, también visto solamente para hombres y es raro ver de repente una mujer que sea mecánica” (E2, p13).

Las percepciones de los (as) estudiantes se unifican en cuanto a la existencia de prejuicios y estigmatizaciones a nivel consciente o inconsciente dependiendo de la carrera por la cual se opte al margen de que se pueda ejercer la profesión que se desea dependiendo de las capacidades cognitivas de cada persona.

"Si a un hombre, por ejemplo, no sé le gustara estudiar danza o incluso ballet (...), igual inconscientemente la gente lo ve como extraño. Igual que una mujer que juega futbol, pero Yo creo que de a poco se va a ir como soltando más ese estigma por así decirlo" (E3, p13). "No debiese pero si existen, por ejemplo párvulo, párvulo por ejemplo la mayoría son mujeres y el hombre que entra ahí es como muy mal visto y la mayoría de las veces yo he visto que son niños gay, mecánica también es como también visto solamente para hombres y es raro ver de repente una mujer que sea mecánica me entiende "(E2, p13).

Asimismo la división ha legitimado una valoración desigual entre hombres y mujeres, subvalorando a estas últimas y entregando un mayor prestigio social y valor económico para el hombre en los casos en los que la mujer participa en las actividades públicas, su presencia a juicio de los (as) estudiantes aún no se produce en plena igualdad con los hombres, es decir, el hecho de ser mujer se traduce en el aspecto laboral en inferiores condiciones económicas. En este sentido, los (as) estudiantes manifiestan que al margen que el trabajo que realice la mujer, incluso si es el mismo que realiza el hombre, la mujer recibe un menor salario. Esto lo asocian a distintos factores, entre ellos culturales por ejemplo, el machismo de que el hombre tiene mayor capacidad y por lo tanto debe ganar más dinero, en otros casos al tema del embarazo y las "posibles" ausencias que ello pueda implicar en el caso de enfermedad del hijo etc.

No obstante lo anterior, los (as) estudiantes manifiestan que si bien esta realidad aún persiste, presenta matices con el factor educativo. En este sentido, visibilizan la educación como una variable clave para la igualdad y equidad de género, es en ella donde observan que se producen más oportunidades igualitarias y en la cual el sexo biológico no marca desigualdades, en lo que 
respecta a los requisitos de ingreso, en donde lo que importa es el puntaje, en el acceso a becas en donde cuenta la situación económica, etc. Además de visibilizarla como el medio a través del cual se accede a una igualación de oportunidades en el mercado laboral. Ante esta evaluación, ellos visibilizan la importancia y necesidad de incorporar la perspectiva de género en la formación docente inicial, pues como futuros educadores de las nuevas generaciones necesitan tener las bases teóricas y metodológicas para modificar concepciones erróneas respecto de la diferenciación existente entre hombre y mujer de forma decisiva.

\section{CONCLUSIONES}

La interpretación de las distintas categorías permitió analizar las percepciones que tienen los (as) estudiantes de pedagogías en relación a la equidad e igualdad de género. En este sentido lo primero que se realizo fue develar la comprensión del término género, el cual es entendido a partir de la diferenciación sexual construida por la sociedad, reforzada por la familia y por las experiencias personales, con lo cual se elaboran ideas de lo que deben ser y hacer hombres y mujeres. Este establecimiento social de prácticas, comportamientos y representaciones fija estereotipos de lo que se espera para cada uno de los sexos, iniciándose con ello una serie de desigualdades en los distintos ámbitos de la vida.

Uno de ellos es el ámbito doméstico en el cual si bien existe una apertura a compartir las actividades entre ambos sexos, la mujer continúa siendo la encargada principal de las labores del hogar y del cuidado de los hijos. De ahí que la maternidad y los beneficios legales sean percibidos por los (as) estudiantes como uno de los principales obstáculos para que la mujer pueda acceder y permanecer en igualdad de condiciones que los hombres en el mercado laboral. En este sentido y pese a los avances en la legislación laboral en Chile, esta continua asignando el cuidado de los hijos principalmente a las mujeres, por lo cual, más que protegerla se convierte en un obstáculo para su incorporación en el mercado laboral, a diferencia de lo que ocurre con los hombres que reciben la función primordial de proveedores y se liberan parcial o totalmente de las tareas y actividades asociadas al trabajo doméstico (Lupica, 2015).

En esta línea y en concordancia a lo revelado por diversos estudios persiste la creencia social de que existen "ciertas" carreras para hombres y "ciertas" carreras para mujeres (Dardón, 2006; Graña, 2008; Navarro Guzmán y Casero Martínez, 2012; Schurch Santana, 2013), las ingenierías son consideradas socialmente como "propias" del sexo masculino, mientras que educación parvularia del sexo femenino. Esta diferenciación de roles de acuerdo al sexo biológico, ha impedido históricamente el desarrollo de las mujeres en igualdad de condiciones que los hombres (Lamas, 1996; Rodríguez et al., 2009; Lupica, 2015). No obstante esta desigualdad disminuye a juicio de los (as) estudiantes por el factor educativo. En este sentido, los participantes reconocen que la educación es el ámbito que brinda más oportunidades en la práctica, para la igualdad y equidad de género. Asimismo, consideran que las diferencias salariales entre mujeres y hombres en el mercado laboral se debilitan cuando existe un nivel educativo acorde a las funciones del cargo en el caso de las mujeres.

Considerando la importancia atribuida al factor educativo en la equidad e igualdad de género, resulta pertinente mencionar la necesidad expuesta por los entrevistados de incorporar la perspectiva de género en la malla curricular de la formación inicial de profesores. Más aún cuando el rol del profesorado es clave en la deconstrucción de las pautas culturales tradicionales de lo femenino y masculino (Vélez Londoño, 2006; García-Pérez, 2011), por lo tanto es necesario que los profesores junto con tener las bases teóricas de lo que es sexo, género, rol de género, identidad de género, etc., esté formado y sensibilizado sobre la desigualdad de género en el mundo y sus consecuencias. Más aún cuando en el caso de Chile estos temas han sido abordados en el plan de estudios solo voluntariamente por el profesorado más sensibilizado con el tema.

En consecuencia y a la luz de los resultados es urgente replantearse la responsabilidad social que poseen las instituciones educativas y en particular la universidad como encargadas de formar a profesionales con poder de intervención y toma de decisiones. Asimismo es un llamado para revisar los planes de estudios vigentes, en especial en el caso de Chile, que 
parecen no haber logrado comprender que un curriculum que incorpora la perspectiva de género impacta tanto a mujeres como a hombres, y beneficia al conjunto de la sociedad, al levantar obstáculos y discriminaciones, y al relevar a los hombres de muchos supuestos de género que son también un peso y una injusticia. Finalmente, creemos necesario re-significar el rol de las universidades como espacios públicos donde se construyen conocimientos, se genera reflexión, se investiga en torno a problemáticas de la realidad y se desarrollan estrategias de acción social democráticas para la transformación de los espacios públicos y privados.

\section{BIBLIOGRAFÍA}

Abett de la Torre Díaz, Paloma (2014), "Educación y género: una reflexión sobre los principios de igualdad y diversidad en la educación chilena en el escenario de la reforma educativa actual", Paulo Freire. Revista de Pedagogía Crítica, núm. 16, pp. 35-47.

Montecino, Sonia y Alexandra Obach (1999), Género y epistemología: mujeres y disciplinas, Chile, Lom Ediciones.

Barrios, Lorena (2011), "La mujer en la televisión: el caso chileno", Cuadernos de información, núm. 29, pp. 105-116.

Butler, Judith (1997), "Sujetos de sexo/género/deseo", Revista Feminaria, vol. 10, núm.19, pp. 109-125.

Bourdieu, Pierre (2000), La dominación masculina, Barcelona, Anagrama.

Burin, Mabel y Irene Meler (1998), Género y familia: poder, amor y sexualidad en la construcción de la subjetividad. Buenos Aires, Paidós.

Cardona Moltó, María Cristina (2002), Introducción a los métodos de investigación educativa. Madrid, EOS.

Caviedes, Elizabeth, Alejandra Barrientos y Patricia Fernández (2006), "La desigualdad olvidada: género y educación en chile”, Equidad de género, pp. 99-148.

Conde, María Rosa, Mercedes del Hoyo Hurtado (2011), "La mujer y el hombre en la publicidad televisiva: imágenes y estereotipos", Zer-Revista de Estudios de Comunicación, vol. 11, núm. 21, pp. 161-175.

Dardón, María Sandra, Elizabeth Verde Flota, Georgina Gallardo Hernández, Silvia Tamez González, Guadalupe Delgado Sánchez y Luis Ortiz-Hernández (2006), "Diferencias entre hombres y mujeres respecto a la elección de carreras relacionadas con atención a la salud”, Revista de estudios de género. La Ventana, Vol. 3, núm. 24, pp. 204-228.

Departamento de estudios laborales; Departamento de estudios sociales; Instituto nacional de estadística (2015), Mujeres en chile y mercado del trabajo. Participación laboral femenina y brechas salariales. Chile: Instituto Nacional de Estadística.

Díaz, María Isabel (1999), “Algunas reflexiones acerca de: la dimensión de género en el curriculum de la educación parvularia”, Temas Pedagógicos. Serie de Cuadernillos de Estudio, pp. 1-21.

Fajardo, Elena (2007), "Construcción de género y ficción televisiva en España", Comunicar: Revista científica iberoamericana de comunicación y educación, núm. 28, pp. 229-236. 
Flores, Raquel (2007), "Representaciones de género de profesores y profesoras de matemática, y su incidencia en los resultados académicos de alumnos y alumnas", Revista iberoamericana de educación, núm. 43, pp. 103-118.

García-Pérez, Rafael, María Ángeles Rebollo, Luisa Vega, Raquel Barragán-Sánchez, Olga Buzón y Joaquín Piedra (2011), "El patriarcado no es transparente: competencias del profesorado para reconocer desigualdad”, Cultura y Educación, vol. 23, núm. 3, pp. 385-397.

Glaser, Barney y Anselm Strauss (1967), The discocovery of grounded theory: Strategies for qualitative research, Chicago IL, Aldine.

Godoy, Lorena y Antonio Mladinic (2009), "Estereotipos y roles de género en la evaluación laboral y personal de hombres y mujeres en cargos de dirección", Psykhe (Santiago), vol. 18, núm. 2, pp. 51-64.

Gómez, María, Antonio García y Beatriz Vicario (2015), "Indicadores de violencia de género en las relaciones amorosas. Estudio de caso en adolescentes chilenos", Pedagogía social. Revista interuniversitaria, núm. 26, pp. 85-109.

Gore, Jennifer (1996), Controversias entre las pedagogías: discursos críticos y feministas como regímenes de verdad, La Coruña Madrid, Fundación Paideia Morata.

Graña, François (2008), "El asalto de las mujeres a las carreras universitarias "masculinas": cambio y continuidad en la discriminación de género", PRAXIS educativa, vol. 12, núm. 12, pp. $77-86$.

Guerrero, Elizabeth, Victoria Hurtado, Ximena Azúa y Patricia Provoste (2006), "Material de Apoyo con perspectiva de género para formadores y formadoras", Chile: Santiago, Ministerio de Educación, Hexagrama, Consultoras y CPEIP, pp. 1-124.

Lagarde, Marcela (1996), Género y feminismo. Desarrollo humano y democracia, Madrid, Horas y Horas.

Lamas, Marta (1996), El género. La construcción cultural de la diferencia sexual, México: Miguel Ángel Porrúa, PUEG-UNAM.

Lamas, Marta (2015), "Usos, dificultades y posibilidades de la categoría género", Revista de estudios de género. La ventana, núm. 1, pp. 10-61.

López-Navajas, Ana (2014), "Análisis de la ausencia de las mujeres en los manuales de la ESO: una genealogía de conocimiento ocultada", Revista de educación, núm. 363, pp. 282-308.

Vélez Londoño, Claudia (2006),"La educación: una opción para erradicar la discriminación de género", Transatlántica de educación, núm. 6, pp. 79-87.

Lupica, Carina (2015), "Corresponsabilidad de los cuidados y autonomía económica de las mujeres: lecciones aprendidas del Permiso Postnatal Parental en Chile", Cepal, núm. 126, pp. 149.

Méndez, Griselda (2014), "Habitus, estereotipos y roles de género. Percepciones de profesores y estudiantes”, Revista Docencia Universitaria, vol. 14, núm. 1, pp. 89-105.

Moreno, Amparo (1986), El arquetipo viril protagonista de la historia, Barcelona, La Sal. 
Navarro Guzmán, Capilla y Antonio Casero Martínez (2012), “Análisis de las diferencias de género en la elección de estudios universitarios", Estudios sobre educación, Vol. 22, pp. 115132.

Rebolledo, Loreto (2015), "Impacto de los estudios de género en la democratización de las instituciones de educación superior", Revista de Estudios de Género. La Ventana, vol. 2, núm. 13 , pp. 75-93.

Richter, Jacqueline (2007), "Segmentadas y segregadas: las mujeres en la fuerza de trabajo en Venezuela", Politeia, vol. 30, núm. 39, pp.151-185.

Rodríguez Teijeiro, Domingo, María José Martínez Patiño y Covadonga Mateos Padorno (2009), "Identidad y estereotipos de la mujer en el deporte: una aproximación a la evolución histórica", Revista de Investigación en Educación, núm. 2, pp. 109-126.

Ruiz Olabuénaga, José (2007), Metodología de Investigación Cualitativa, Bilbao, Universidad de Deusto.

Rubio-Garay, Fernando, Miguel Carrasco, Pedro Amor y María López-González (2015), "Factores asociados a la violencia en el noviazgo entre adolescentes: una revisión crítica", Anuario de Psicología Jurídica, núm. 25, pp. 47-56.

Scott, Joan (2008) Género e historia, México, Fondo de Cultura Económica, UACM.

Schurch Santana, Roberto (2013), "El retorno de las carreras: un estudio de caso de los factores que inciden en las remuneraciones de universitarios recién titulados", Calidad en la educación, núm. 38, pp. 215-244.

Stoller, Robert (1968), Sex and gender, New York, Science House.

Strauss, Anselm y Juliet Corbin (2002), Bases de la investigación cualitativa: técnicas y procedimientos para desarrollar la teoría fundamentada, Colombia, Editorial Universidad de Antioquía.

Soto-Quevedo, Osvaldo (2015), "Rol del sexismo ambivalente y de la transgresión de estereotipo de género en la atribución de culpa a mujeres víctimas de violencia de pareja", Acta Colombiana De Psicología, vol. 15, núm. 2, pp. 135-147, en: http://editorial.ucatolica.edu.co/ojsucatolica/revistas ucatolica/index.php/acta-colombianapsicologia/article/view/273 (consulta 8 de marzo 2016).

Valdivia, Maruzzella y Luis González (2014), "Violencia en el noviazgo y pololeo: una actualización proyectada hacia la adolescencia", Revista de Psicología (PUCP), vol. 32, núm. 2, pp. 329-355. 